\title{
Analysis of Resource Sharing Model of Civil-military Integration Based on the Evolutionary Game Theory
}

\author{
Xiang Sun ${ }^{1, a}$, Jie Zhang ${ }^{2, b}$ and S.Xu Z $^{3, c}$ \\ ${ }^{1}$ Hefei University of Technology, Hefei,China \\ ${ }^{2}$ Hefei University of Technology, Hefei,China \\ ${ }^{3}$ Hefei University of Technology, Hefei,China \\ asunxiang69@hfut.edu.cn, bj199301@163.com, 'xssx69@163.com
}

Keywords: Civil-military Integration,Resource Sharing,Evolutionary Game Model

Abstract. The paper based on "limited rationality" and "replication dynamics" ideas of evolutionary game theory, assumes that military enterprise and civil enterprise prefer to adopt a fixed strategy, establishes an evolutionary game model of resource sharing to analyze the key factors affecting the relationship of resource sharing in civil-military integration, and tries to find the evolutionary path and equilibrium strategy of the cooperative game between military enterprise and civil enterprise.

\section{Introduction}

With the development of science and technology, how to meet the military needs and promote the rapid growth of the national economy at the same time has become a key issue to be solved, the fundamental way out is to achieve civil-military integration, then promote military enterprise and civilian enterprise to achieve resources sharing of economic, technology, personnel and so on.

Chen Bai, Jie Peng and Jinbing $\mathrm{Li}$ construct the game model between the supplier and the intermediary in the information resource sharing and solve it, analyze the model variables, then propose strategies to promote the sharing of information ${ }^{[1]}$. Haiou Liu, Yaming Zhang from the perspective of cooperative innovation game, establish the "reputation game" model of science and technology resources sharing in Beijing, Tianjin and Hebei, to seek the breakthrough point of Nash equilibrium, and finally put forward the countermeasures and suggestions on promoting the sharing of science and technology resources in Beijing, Tianjin and Hebei ${ }^{[2]}$. Lanjie Chen, Yanlin Liu point out obstacles of government information resources sharing in Beijing, Tianjin and Hebei through the comparison and analysis of the current situation of domestic and international regional government information resources sharing ${ }^{[3]}$. Han Wang from the perspective of realizing the sharing of military and civilian information resources, propose to speed up the military information coordination department construction and improve the military integrated information communication platform, so as to improve the quality of civil-military information resources widely shared ${ }^{[4]}$.

Most of the existing researches are carried out in the construction of resource sharing mechanism and propose some countermeasures and suggestions, quantitative researches are less. In this paper, we will start from the perspective of military enterprises and civil enterprises, breaking through the traditional classical game theory, based on the limited rationality ideas, then build the evolution game model of civil-military integration resources to analyze the evolutionary path and equilibrium strategy of the cooperation game between military enterprise and civil enterprise .

\section{Modeling}

Model Selection.The classical game theory has great theoretical advantages in explaining many economic problems, but it has three problems: The first one is the assumption that the main body of the behavior has a perfect rational thinking, always aim to maximize their own interests. But in reality, due to the closure and monopoly of military industry, it will inevitably lead to poor information channels, resulting in military and civilian enterprises can not always have a perfect judgment and prediction ability; The second one is the "common knowledge" hypothesis of both 
players, all the participants are rational and know that other participants are rational too. Obviously, this assumption is often not guaranteed in civil-military integration; The third one is the choice of multiple Nash equilibrium, in most cases, the classical game theory has more than one Nash equilibrium, it is difficult to determine which one is the game's rational results. In order to solve these problems, many economists have made a lot of attempts over the years, and developed many branches of Game Theory.

In many branches of Game Theory, evolutionary game theory has certain advantages in solving the above problems. It combines the analysis of game theory with dynamic evolution process analysis, the emphasis is a dynamic equilibrium which conforms to the process of civil-military integration. It model man as a limited rational game party and do not require the condition of complete information, this is in accord with the situation that information flow is not smooth between the civilian and military industry. It insists that human is usually by trial and error method to reach game equilibrium, and the selected equilibrium is the function of equilibrium process has been reached. The strategies of players are not only determined by the Personal rationality, but also by the historical factors, institutional factors, social customs, practices and certain details of the equilibrium process will have an impact on the selection of multiple equilibrium of the game. It assumes that there are many participants in a system, game parties are random sampling from the collection of participants in each round, repeated many rounds. This is consistent with the cooperation mechanism of military enterprise and civilian enterprise. It provides the standard to select the final equilibrium results from the multiple Nash equilibrium and describes the dynamic process of equilibrium realization.

Model Hypothesis.From the economic point of view, the resource sharing of military and civilian enterprises is a choice of the economic efficiency of the resources. If a player considers that the benefit of the exclusive use of resources is greater than the benefit of sharing the resources, the enterprise may seek the exclusive right to use the resources, otherwise, the enterprise needs to seek effective ways to share. This shows that the decision depending on whether the expected benefits are greater than the expected costs if the exclusive resources are abandoned. From the perspective of enterprise income, we try to establish a resource sharing model through the game analysis. The construction of model based on the following hypothesis.

Hypothesis 1 In the process of resource sharing, it only involves two kinds of decision makers, military enterprises and civil enterprises, their strategies are (resource sharing, non resource sharing).

Hypothesis 2 In the process of resource sharing, the income of two kinds of decision-making body is made up of two parts: one is the normal income that is obtained without sharing resources, the other is the shared benefits achieved by means of absorbing, digesting, learning, learning from each other.

Hypothesis 3 Because of the limitation of the system and other reasons, it is possible for game parties to have opportunistic behaviors which damage the interests of the other party in exchange for their own interests.

\section{Inference Analysis}

Cooperative Game Payoff Matrix.According to the hypothesis 2, the income of the two parties in the process of sharing resources in civil-military integration is made up of normal income and the shared benefits, the total amount of shared benefits is limited by the size of the resources which the two parties are willing to invest and the shared rate of return, and the shared benefits obtained by the respective is dependent on the distribution rates of the total amount of shared benefits. If $f$ indicates the size of resources that the players are willing to invest in, $r$ indicates the shared rate of return, then the total amount of shared benefits is $r f$. In the case of non resource sharing, the normal income of military enterprise is $a_{1}$, the normal income of civilian enterprises is $a_{2}$. In the case of resource sharing, the proportion of shared benefits of civilian enterprise is $q$, the proportion of shared benefits of military enterprise is 1-q, then the total income of the civilian enterprises in the resource sharing process is $a_{2}+q r f$, the total income of the military enterprises in the resource sharing process is 
$a_{1}+(1-q) r f$. In order to achieve resource sharing, military enterprise and civilian enterprise also need a certain capital, technology and other input costs, the corresponding investment costs are $c_{1} f_{1}$ and $c_{2} f_{2}$, where $f_{1}$ indicates the investment scale of military enterprise shared resources, $f_{2}$ indicates the investment scale of civilian enterprise shared resources, $c_{1}$ is the cost coefficient of the military enterprises and $c_{2}$ is the cost coefficient of the civil enterprises. Due to the existence of opportunism, there is a certain risk factor $l$ when people take the behavior of sharing, if civilian enterprise to choose the "resource sharing" strategy, military enterprise to choose the "non resource sharing" strategy, the two sides of the proceeds will be: $a_{2}-c_{2} f_{2}$ and $a_{1}+l_{1} f_{2}$, if military enterprise to choose the "resource sharing" strategy, civilian enterprise to choose the "non resource sharing" strategy, the two sides of the proceeds will be: $a_{1}-c_{1} f_{1}$ and $a_{2}+l_{2} f_{1}$, where $l_{1} f_{2}$ and $l_{2} f_{1}$ were opportunistic earnings of both enterprises. The cooperative game payment matrix is shown in Table 1.

Table 1. the cooperative game payment matrix of the civil enterprise and military enterprise

\begin{tabular}{|c|c|c|c|}
\hline Game parties & \multicolumn{3}{|l|}{ Civil enterprise } \\
\hline \multirow{3}{*}{$\begin{array}{l}\text { Military } \\
\text { enterprise }\end{array}$} & Policy space & Resource sharing & Non resource sharing \\
\hline & Resource sharing & $a_{1}+(1-q) r f-c_{1} f_{1}: a_{2}+q r f-c_{2} f_{2}$ & $a_{1}-c_{1} f_{1}: a_{2}+l_{2} f_{1}$ \\
\hline & Non resource sharing & $a_{1}+l_{1} f_{2}: a_{2}-c_{2} f_{2}$ & $a_{1}: a_{2}$ \\
\hline
\end{tabular}

Inference Calculation of Evolutionary Game Model.We define the probability of selecting the "resource sharing" strategy for military enterprise is $w^{J}$, then the probability of selecting the "non resource sharing" strategy for military enterprise is $1-w^{J}$, when taking "resource sharing" strategy or "non resource sharing" strategy, the gains obtained by military enterprise are represented by $U_{g}^{J}$ and $U_{f}^{J}$ respectively, the expected benefits are expressed as $U^{J}$; the probability of selecting the "resource sharing" strategy for civil enterprise is $w^{M}$, then the probability of selecting the "non resource sharing" strategy for military enterprise is $1-w^{M}$, when taking "resource sharing" strategy or "non resource sharing" strategy, the gains obtained by military enterprise are represented by $U_{g}^{M}$ and $U_{f}^{M}$ respectively, the expected benefits are expressed as $U^{M}$.

The income of selecting the "resource sharing" strategy for civil enterprise is:

$$
U_{g}^{M}=w^{J}\left(a_{2}+q r f-c_{2} f_{2}\right)+\left(1-w^{J}\right)\left(a_{2}-c_{2} f_{2}\right)=a_{2}-c_{2} f_{2}+w^{J} q r f .
$$

The income of selecting the "non resource sharing" strategy for civil enterprise is:

$U_{f}^{M}=w^{J}\left(a_{2}+l_{2} f_{1}\right)+\left(1-w^{J}\right) a_{2}=a_{2}+w^{J} l_{2} f_{1}$.

So,the expected benefits for civil enterprise is:

$$
\begin{aligned}
U^{M} & =w^{M} U_{g}^{M}+\left(1-w^{M}\right) U_{f}^{M}=w^{M}\left[a_{2}-c_{2} f_{2}+w^{J} q r f\right]+\left(1-w^{M}\right)\left(a_{2}+w^{J} l_{2} f_{1}\right) \\
& =a_{2}-w^{M} c_{2} f_{2}+w^{M} w^{J} q r f+\left(1-w^{M}\right) w^{J} l_{2} f_{1}
\end{aligned}
$$

The income of selecting the "resource sharing” strategy for military enterprise is:

$$
U_{\mathrm{g}}^{J}=w^{M}\left[a_{1}+(1-q) r f-c_{1} f_{1}\right]+\left(1-w^{M}\right)\left(a_{1}-c_{1} f_{1}\right)=a_{1}-c_{1} f_{1}+w^{M}(1-q) r f .
$$

The income of selecting the "non resource sharing" strategy for military enterprise is:

$$
U_{f}^{J}=w^{M}\left(a_{1}+l_{1} f_{2}\right)+\left(1-w^{M}\right) a_{1}=a_{1}+w^{M} l_{1} f_{2} .
$$


So,the expected benefits for military enterprise is:

$$
\begin{aligned}
U^{J} & =w^{J} U_{g}^{J}+\left(1-w^{J}\right) U_{f}^{J}=w^{J}\left[a_{1}-c_{1} f_{1}+w^{M}(1-q) r f\right]+\left(1-w^{J}\right)\left(a_{1}+w^{M} l_{1} f_{2}\right) \\
& =a_{1}-w^{J} c_{1} f_{1}+w^{J} w^{M}(1-q) r f+\left(1-w^{J}\right) w^{M} l_{1} f_{2}
\end{aligned}
$$

Dynamic Replication Equation.According to the evolutionary game theory, the dynamic replication equations of the civil and military enterprises are constructed as follows:

$$
\begin{aligned}
& F\left(w^{M}\right)=w^{M}\left(U_{g}^{M}-U^{M}\right)=w^{M}\left(1-w^{M}\right)\left(w^{J} q r f-c_{2} f_{2}-w^{J} l_{2} f_{1}\right) . \\
& H\left(w^{J}\right)=w^{J}\left(U_{g}^{J}-U^{J}\right)=w^{J}\left(1-w^{J}\right)\left[w^{M}(1-q) r f-c_{1} f_{1}-w^{M} l_{1} f_{2}\right] .
\end{aligned}
$$

The evolution process of resource sharing in civil-military integration can be described by the evolution system represented by two dynamic replication equations. According to the method proposed by Friedman, the stability of the evolutionary system can be obtained by analyzing the Jacobi matrix of the evolution system. The Jacobi matrix of the evolution system about the resource sharing in civil-military integration is shown below:

$$
\begin{aligned}
J & =\left[\begin{array}{ll}
\frac{\partial F\left(w^{M}\right)}{\partial w^{M}} & \frac{\partial F\left(w^{M}\right)}{\partial w^{J}} \\
\frac{\partial H\left(w^{M}\right)}{\partial w^{M}} & \frac{\partial H\left(w^{M}\right)}{\partial w^{J}}
\end{array}\right] \\
& =\left[\begin{array}{cc}
\left(1-2 w^{M}\right)\left(w^{J} q r f-c_{2} f_{2}-w^{J} l_{2} f_{1}\right) & w^{M}\left(1-w^{M}\right)\left(q r f-l_{2} f_{1}\right) \\
w^{J}\left(1-w^{J}\right)\left[(1-q) r f-l_{1} f_{2}\right] & \left(1-2 w^{J}\right)\left[w^{M}(1-q) r f-c_{1} f_{1}-w^{M} l_{1} f_{2}\right]
\end{array}\right] .
\end{aligned}
$$

The Jacobi determinant is:

$$
\text { det } \begin{aligned}
J & =\left(1-2 w^{M}\right)\left(1-2 w^{J}\right)\left(w^{J} q r f-c_{2} f_{2}-w^{J} l_{2} f_{1}\right)\left[w^{M}(1-q) r f-c_{1} f_{1}-w^{M} l_{1} f_{2}\right] \\
& -w^{M} w^{J}\left(1-w^{M}\right)\left(1-w^{J}\right)\left(q r f-l_{2} f_{1}\right)\left[(1-q) r f-l_{1} f_{2}\right]
\end{aligned}
$$

The trace of Jacobi determinant is:

$$
\operatorname{traJ}=\left(1-2 w^{M}\right)\left(w^{J} q r f-c_{2} f_{2}-w^{J} l_{2} f_{1}\right)+\left(1-2 w^{J}\right)\left[w^{M}(1-q) r f-c_{1} f_{1}-w^{M} l_{1} f_{2}\right]
$$

Stability Analysis of Equilibrium Points.According to the Jacobi determinant and trace of Jacobi determinant, the stability analysis results of the game equilibrium point of the evolutionary system are obtained, which are shown in Table 2.

Table 2. stability analysis results of resource sharing evolution system in civil-military integration

\begin{tabular}{|c|c|c|c|}
\hline Equilibrium point & Symbol of a determinant & Symbol of trace & Stability of equilibrium points \\
\hline$w^{\mathrm{M}}=0, w^{\mathrm{J}}=0$ & + & - & ESS \\
\hline$w^{\mathrm{M}}=1, w^{\mathrm{J}}=0$ & + & + & Not stable \\
\hline$w^{\mathrm{M}}=0, w^{\mathrm{s}}=1$ & + & + & Not stable \\
\hline$w^{\mathrm{M}}=1, w^{\mathrm{J}}=1$ & + & - & ESS \\
\hline$w^{\mathrm{M}}=w^{\mathrm{M}}, w^{\mathrm{J}}=w^{\mathrm{s}^{\mathrm{s}}}$ & - & 0 & Saddle point \\
\hline
\end{tabular}

From table 2, we can see that in the five local equilibrium points of resource sharing evolution system, only when $w^{M}$ and $w^{J}$ are both 0 or 1 , it is an evolutionary stable strategy, that is, the system is stable only when military enterprise and civilian enterprise both adopt the "resource sharing"strategy or "non resource sharing"strategy. Fig. 1 is a dynamic evolution diagram of resource sharing system in civil-military integration. As shown in the diagram, the only $\mathrm{O}$ point and $\mathrm{C}$ point are stable and are 
the evolutionary stable strategy (ESS), they correspond to the two modes of the enterprise(both take "resource sharing" strategy or take the "non resource sharing" strategy). In addition, the system has two unstable local equilibrium points $\mathrm{A}$ and $\mathrm{B}$ and has a saddle point D. Fig.1 describes the dynamic process of game of two unstable equilibrium points A and B. The broken line connected by A and B and saddle point $\mathrm{D}$ is the critical line of the system to converge to different states, in the right upper part of the broken line, that is the ADBC part, the game system converges to "resource sharing" strategy, and in the left lower part of the broken line, that is the ADBO part, the game system converges to "non resource sharing" strategy. Taking into account the evolution of the system is a long process, many enterprises in the system have maintained the coexistence of resource sharing and non sharing situation.

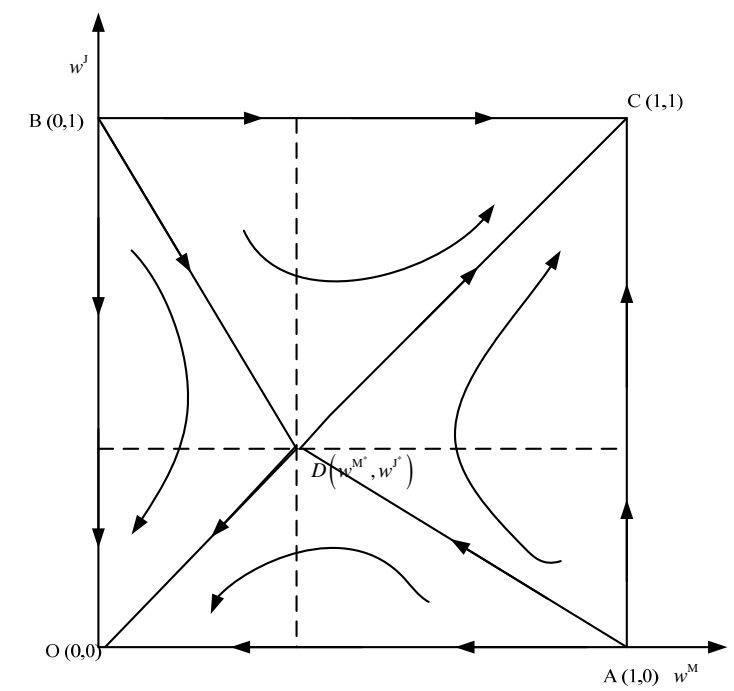

Fig.1.dynamic evolution diagram of resource sharing system in civil-military integration

\section{Conclusion}

In this paper,we analyze the strategy selection problem of "resource sharing" and "non resource sharing" of military enterprise and civil enterprise in the long-term game through the establishment of evolutionary game model. Finally, it is concluded that the long-term equilibrium structure of the resource sharing game system between military enterprise and civil enterprise is likely to be completely shared, and it may be completely non shared, Which path is the path, What kind of state to reach in the end, these are closely related to the payoff matrix and the initial state of the game. That is to say, in the process of the game, the initial values and the change of some parameters of the payoff function of the two sides will lead to the evolution system converge to different equilibrium points.

\section{References}

[1] Chen Bai, Jie Peng, Jinbing Li: The Relationship between Supply and Intermediary of Information Resource Sharing Based on the Game Theory, Science and Technology Management Research, 2014 No.18, p.243 "In Chinese”

[2] Yaming Zhang, Haiou Liu: Technology Resource Sharing Model and Strategy of Beijing,Tianjin and Hebei from the Outlook of Collaborative Innovation Game Theory, China Science and Technology Forum, 2014-01 No.01, p.34 "In Chinese”

[3] Lanjie Chen,Yanlin Liu: Research on the Promoting Mechanism of Government Information Resources Sharing in Beijing,Tianjin and Hebei Region, Information Science, 2015-06 No.6 Vol.33, p.109 "In Chinese”

[4] Han Wang: Discussion on the sharing of military and civilian information resources, Military Economic Research, 2012-01, p.12 “In Chinese”. 